Tôhoku Math. Journ.

31 (1979), 537-547.

\title{
COMPACT COMPLEX SURFACES CONTAINING GLOBAL STRONGLY PSEUDOCONVEX HYPERSURFACES
}

\author{
MASAHIDE KATO \\ (Received December 10, 1978, revised February 28, 1979)
}

Let $X$ be a compact complex manifold of dimension $n \geqq 2$. An open subset $N$ of $X$ is called a spherical shell, if $N$ is biholomorphic to

$$
S_{\varepsilon}=\left\{z \in C^{n}: 1-\varepsilon<\|z\|<1+\varepsilon\right\}
$$

for some $\varepsilon, 0<\varepsilon<1$, where $\|z\|$ denotes the standard complex Euclidean norm of a vector $z=\left(z_{j}\right)$ in the $n$-dimensional complex vector space $C^{n}$, i.e., $\|\boldsymbol{z}\|^{2}=\sum_{j=1}^{n}\left|z_{j}\right|^{2}$. $\quad N$ is called a global spherical shell (abbrev., GSS), if $X-N$ is connected. In [4], we have proved that a compact complex manifold containing a GSS is biholomorphic to a deformation of a modification of a primary Hopf manifold at finitely many points. In this paper we restrict ourselves to the case of surfaces, i.e., $n=2$, and consider compact complex surfaces containing (real analytic) global strongly pseudoconvex hypersurfaces (GSPH) which bound Stein domains possibly with finitely many isolated singular points. Then we can determine all such surfaces (Theorem).

Here we shall use the definitions and some results in Rossi [5, 6]. Let $\Sigma$ be a compact real analytic CR-hypersurface with $\operatorname{dim}_{R} \Sigma=3$. It is known that $\Sigma$ admits a realization as a real hypersurface in a complex manifold of (complex) dimension 2. Namely, there exist a complex manifold $M$ of dimension 2 and a CR-injection $j: \Sigma \rightarrow M$ such that the CR-structure on $\Sigma$ coincides with the CR-structure induced from $M$. Moreover, if $j_{i}: \Sigma \rightarrow M_{i}(i=1,2)$ are two realizations of $\Sigma$, then $j_{2} \circ j_{1}^{-1}$ extends to a biholomorphic mapping between small neighborhoods of $j_{i}(\Sigma)$ ([6]). This implies that the realization of $\Sigma$ is unique as a germ.

We say that $\Sigma$ bounds a Stein domain, if there exist a (reduced irreducible) complex space $\hat{M}$, a subdomain $M$ of $\hat{M}$ which is free from singular points, and a realization $j: \Sigma \rightarrow M$ of $\Sigma$, such that $j(\Sigma)$ bounds a relatively compact Stein open subset $D$ of $\hat{M}$. Note that $D$ may have finitely many isolated singular points. We remark that there exist strongly pseudoconvex hypersurfaces $\Sigma$ with $\operatorname{dim}_{R} \Sigma=3$ such that $\Sigma$ do not bound any Stein domains ([5]).

Let $S$ be a compact complex manifold of dimension 2, which will be 
called a surface. Let $\Sigma$ be a real analytic submanifold of (real) dimension 3 in $S$. Then $\Sigma$ admits the real analytic CR-structure induced from $S$. Suppose that $\Sigma$ is strongly pseudoconvex.

DEFINITION $1 . \quad \Sigma$ is called a global strongly pseudoconvex hypersurface (GSPH) in $S$, if $S-\Sigma$ is connected.

LEMMA 1 ([5]). Let $\Sigma$ be a strongly pseudoconvex hypersurface realized in a complex manifold $M$. Then there are a neighborhood $N$ of $\Sigma$ and a strongly plurisubharmonic function $\phi$ defined on $N$ such that $\Sigma=\{x \in N: \varphi(x)=0\}$.

Now we shall prove the following

THEOREM. Let $S$ be a compact complex manifold of dimension 2. Then $S$ contains a real analytic GSPH which bounds a Stein domain possibly with finitely many isolated singular points, if and only if $S$ is one of the following:

(i) $S$ contains a GSS,

(ii) $S$ is biholomorphic to a modification of a surface of Class $\mathrm{VI}_{0}$, an elliptic surface of Class $\mathrm{VII}_{0}$, or a non-primary Hopf surface.

REMARK 1. Primary Hopf surfaces belong to (i). Generally, $S$ satisfying the assumption of the theorem belongs to (i), if and only if the fundamental group of $S$ is infinite cyclic.

REMark 2. The "if part" of the theorem is clear, since surfaces without exceptional curves of the first kind which belong to (ii) of the theorem are biholomorphic to submanifolds of Hopf manifolds, and since any submanifold of Hopf manifolds contains real analytic GSPH's [2, 3]. Therefore the rest of the paper is devoted to the proof of the "only if part" of the theorem.

We shall repeat the argument of $\S 1$ [4]. Consider the following homology exact sequence with $\boldsymbol{Z}$-coefficients:

$$
\begin{aligned}
\cdots & \rightarrow H_{1}(S-\Sigma) \rightarrow H_{1}(S) \rightarrow H_{1}(S, S-\Sigma) \\
& \rightarrow H_{0}(S-\Sigma) \rightarrow H_{0}(S) \rightarrow H_{0}(S, S-\Sigma) \rightarrow 0 .
\end{aligned}
$$

By the duality theorem, we have

$$
H_{1}(S, S-\Sigma) \cong H^{3}(\Sigma) \cong Z \text {, and } H_{0}(S, S-\Sigma) \cong H^{4}(\Sigma)=0 \text {. }
$$

Since both $S$ and $S-\Sigma$ are connected by assumption, we have

$$
H_{0}(S-\Sigma) \cong H_{0}(S) \cong Z \text {. }
$$

Therefore (1) is reduced to 


$$
\cdots \rightarrow H_{1}(S-\Sigma) \rightarrow H_{1}(S) \stackrel{p}{\rightarrow} \boldsymbol{Z} \rightarrow 0 .
$$

Let $h: \pi_{1}(S) \rightarrow H_{1}(S)$ be the Hurewicz homomorphism. Put

$$
j=p \circ h: \pi_{1}(S) \rightarrow Z \text {. }
$$

Take the infinite cyclic covering

$$
\tilde{\omega}: \widetilde{S} \rightarrow S
$$

of $S$ such that $\pi_{1}(\widetilde{S})$ is equal to the kernel of $j$. Denote by $\widetilde{g}$ the holomorphic automorphism of $\widetilde{S}$ such that the orbit space $\widetilde{S} /\langle\widetilde{g}\rangle$ is equal to $S$. Let

$$
i: S-\Sigma \rightarrow S
$$

be the inclusion and

$$
i_{*}: \pi_{1}(S-\Sigma) \rightarrow \pi_{1}(S)
$$

the induced homomorphism. From (2),

$$
i_{*} \pi_{1}(S-\Sigma) \subset \operatorname{ker} j
$$

follows. Hence there is a lifting

$$
\tilde{i}: S-\Sigma \rightarrow \widetilde{S}
$$

of $i$ and we obtain the commutative diagram

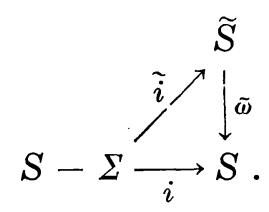

Let $F=\tilde{i}(S-\Sigma)$. Let $N^{\prime}$ be a sufficiently small tubular neighborhood of $\Sigma$ in $S$. By Lemma 1, we can assume that there is a strongly plurisubharmonic function $\varphi$ on $N^{\prime}$ such that

$$
\Sigma=\left\{x \in N^{\prime}: \varphi(x)=0\right\} \text {. }
$$

Take a small $\varepsilon>0$ such that

$$
N(\varepsilon)=\left\{x \in N^{\prime}:|\varphi(x)|<\varepsilon\right\}
$$

is relatively compact in $N^{\prime}$. Put

$$
\begin{aligned}
& N^{+}(\varepsilon)=\left\{x \in N^{\prime}: 0 \leqq \varphi(x)<\varepsilon\right\}, \text { and } \\
& N^{-}(\varepsilon)=\left\{x \in N^{\prime}:-\varepsilon<\varphi(x) \leqq 0\right\} .
\end{aligned}
$$

There are exactly two connected components $N_{1}(\varepsilon), N_{2}(\varepsilon)$ of $\tilde{\omega}^{-1}(N(\varepsilon))$ such that $N_{i}(\varepsilon) \cap F \neq \varnothing(i=1,2)$. Suppose that $\tilde{\omega}\left(N_{2}(\varepsilon) \cap \bar{F}\right)=N^{+}(\varepsilon)$. Then $\tilde{\omega}\left(N_{2}(\varepsilon) \cap F\right) \cap N^{-}(\varepsilon)=\tilde{\omega}\left(N_{1}(\varepsilon) \cap F\right) \cap N^{+}(\varepsilon)=\varnothing$ and $\tilde{\omega}\left(N_{1}(\varepsilon) \cap \bar{F}\right)=N^{-}(\varepsilon)$. 
Taking $\widetilde{g}^{-1}$ instead of $\widetilde{g}$ if necessary, we can assume that $\widetilde{g}\left(N_{1}(\varepsilon)\right)=N_{2}(\varepsilon)$. We put

$$
E(\varepsilon)=N_{1}(\varepsilon) \cup F \cup N_{2}(\varepsilon) .
$$

Let $\Sigma_{i}$ be a connected component of $\tilde{\omega}^{-1}(\Sigma)$ contained in $N_{i}(\varepsilon)$. By assumption $\Sigma_{2}$ bounds a Stein domain. Hence, by the uniqueness of the realization of $\Sigma$, there is a Stein space $B$ and a compact subset $K^{\prime}$ such that $B-K^{\prime}$ is biholomorphic to $N_{2}\left(\delta_{0}\right)$ for a sufficiently small $\delta_{0}, 0<\delta_{0}<\varepsilon$. Let $\eta: B-K^{\prime} \rightarrow N_{2}\left(\delta_{0}\right)$ be the holomorphic mapping. Identifying $x \in B-K^{\prime}$ with $\eta(x) \in N_{2}\left(\delta_{0}\right)$, we obtain a complex space

$$
Z(\varepsilon)=B \cup_{\eta}\left(N_{2}\left(\delta_{0}\right) \cup F \cup N_{1}(\varepsilon)\right) \text {. }
$$

For any $\varepsilon_{1}, 0<\varepsilon_{1} \leqq \varepsilon, Z\left(\varepsilon_{1}\right)$ can be regarded naturally as a subdomain of $Z(\varepsilon)$. Since $E(\varepsilon)$ is non-singular, all singular points of $Z(\varepsilon)$ are contained in $K^{\prime}$ and they are isolated. Moreover we can assume that $Z(\varepsilon)$ is normal. Let $0<\delta<\delta_{0}$. Since $Z(\varepsilon)$ is strongly pseudoconvex and $B$ is a normal Stein space, the holomorphic mapping

$$
\widetilde{g} \mid N_{1}(\delta): N_{1}(\delta) \rightarrow N_{2}(\delta)
$$

induced by $\widetilde{g}$ extends to a holomorphic mapping

$$
g: Z(\delta) \rightarrow Z(\delta)
$$

by a theorem of Hartogs. It is easily checked that $g(Z(\delta))=D(\delta)$, where we put $D(\delta)=K \cup N_{2}(\delta)$, and $K=B-F$.

Lemma 2. There exists a point $O^{*} \in K$ such that

$$
\bigcap_{\nu \geq 1} g^{\nu}(Z(\delta))=\left\{O^{*}\right\} .
$$

The proof is the same as that of Lemma 1 [4].

Let $A$ be the union of all 1-dimensional compact subvarieties in $Z(\delta)$. Then $A$ is called the maximal compact analytic subset in $Z(\delta)$. We see easily that, for $0<\delta_{1}<\delta$, the maximal compact analytic subset in $Z\left(\delta_{1}\right)$ coincides with $A$ provided that $\delta-\delta_{1}$ is sufficiently small.

In the following, we shall consider two cases $(\alpha) O^{*} \notin A$, and $(\beta) O^{*} \in A$, separately.

( $\alpha$ ) Case $O^{*} \notin A$ (including the case $A=\varnothing$ ).

Proposition 1. If $O^{*} \notin A$, then $S$ is a modification of a surface of Class $\mathrm{VI}_{0}$, an elliptic surface of Class $\mathrm{VII}_{0}$, or a Hopf surface.

Proof. By using the same method as in $[4, \S 2]$ and $[3$, p. 560], we can construct a proper holomorphic mapping of degree 1 onto a sub- 
variety of a higher dimensional Hopf manifold. It is easy to see that the singularities of the image variety must be isolated. Hence we infer that the image is non-singular, since no positive dimensional subvarieties of a Hopf manifold admit isolated singularities (cf. [2]). It is shown in [2] that a 2-dimensional submanifold is one of the above.

q.e.d.

REMARK 3. If $O^{*} \notin A$ and if $O^{*}$ is a non-singular point, then $S$ is a modification of a primary Hopf surface. If $O^{*} \notin A$ and if $O^{*}$ is a singular point, then $S$ is a modification of a surface of Class $\mathrm{VI}_{0}$, an elliptic surface of Class $\mathrm{VII}_{0}$, or a non-primary Hopf surface.

( $\beta)$ Case $O^{*} \in A$.

Let

$$
\lambda: Z^{*}(\delta) \rightarrow Z(\delta)
$$

be the simultaneous minimal resolutions of all singular points of $Z(\delta)$. Then $g: Z(\delta) \rightarrow Z(\delta)$ induces a holomorphic mapping

$$
g^{*}: Z^{*}(\delta) \rightarrow Z^{*}(\delta)
$$

such that

$$
\lambda \circ g^{*}=g \circ \lambda .
$$

Let $A_{0}$ be the connected component of $A$ containing $O^{*}$. We put $A^{*}=$ $\lambda^{-1}\left(A_{0}\right)$ and $B^{*}=\lambda^{-1}\left(O^{*}\right)$.

LEMma 3. There exists a positive integer $\nu_{0}$ such that $g^{*^{\nu}}\left(A^{*}\right)$ is a point for all $\nu \geqq \nu_{0}$.

Proof. Since $g\left(A_{0}\right)=O^{*}$, it is clear that

$$
g^{*^{\nu}}\left(A^{*}\right) \subset B^{*}
$$

for all $\nu \geqq 1$. Put $B_{1}^{*}=\bigcap_{\nu \geqq 1} g^{* \nu}\left(B^{*}\right)$. Then obviously we have $g^{*}\left(B_{1}^{*}\right)=$ $B_{1}^{*}$. Suppose that $B_{1}^{*}$ contains irreducible curves $C_{1}, \cdots, C_{r}$. Since $g^{*}\left(B_{1}^{*}\right)=B_{1}^{*}$, we can classify $C_{i}$ 's into the orbits of $g^{*}$. Let $\left(C_{j 0}, \cdots, C_{j r_{j-1}}\right)(j=1,2, \cdots, s)$ be the orbits, where we can assume that

$$
\left\{\begin{array}{l}
g^{*}\left(C_{j k}\right)=C_{j k+1} \quad\left(k=0, \cdots, r_{j}-1\right) \\
g^{*}\left(C_{j r_{j-1}}\right)=C_{j 0}
\end{array}\right.
$$

Since $A^{*}$ is connected, there exists an orbit, say $\left(C_{10}, \cdots, C_{1 r_{0}-1}\right)$, such that $C_{1 j}$ intersects a curve $C$ which contracts to a point by $g^{*}$. We can assume that $C_{1 j}=C_{10}$. Then we have

$$
C_{10}{ }^{2}<C_{11}{ }^{2} \text {. }
$$

On the other hand, by (5), we have 


$$
{C_{10}}^{2} \leqq C_{11}{ }^{2} \leqq{C_{12}}^{2} \leqq \cdots \leqq C_{1 r_{0}-1}{ }^{2} \leqq C_{10}{ }^{2},
$$

which contradicts (6). Hence we infer that $B_{1}^{*}$ consists of points. Since $g^{*}\left(B^{*}\right) \subset B^{*}$ and $B^{*}$ is connected, we conclude that there is a positive integer $\nu_{0}$ such that $g^{* \nu}\left(B^{*}\right)$ consists of a point for all $\nu \geqq \nu_{0}$ Since $g^{*^{\nu}}\left(A^{*}\right)=$ $g^{* \nu}\left(B^{*}\right)$ for all $\nu \geqq \nu_{0}$, we obtain the lemma.

Denote by $O^{* *}$ the point of Lemma 3:

$$
g^{*^{\nu}}\left(A^{*}\right)=\left\{O^{* *}\right\} \quad\left(\nu \geqq \nu_{0}\right) .
$$

Obviously $O^{* *}$ is a point on $B^{*}$.

Lemma 4. $\bigcap_{\nu \geq 1} g^{*^{\nu}}\left(Z^{*}(\delta)\right)=\left\{O^{* *}\right\}$.

Proof. Put

$$
M^{*}=\bigcap_{\nu \geq 1} g^{*^{\nu}}\left(Z^{*}(\delta)\right) .
$$

By Lemma 2 and (3), we have $M^{*} \subset \lambda^{-1}\left(O^{*}\right)=B^{*} \subset A^{*}$. Then, from $M^{*}=g^{*}\left(M^{*}\right)$ and Lemma 3 , it follows that

$$
\left\{O^{* *}\right\} \subset M^{*}=\bigcap_{\nu \geq 1} g^{*^{\nu}}\left(M^{*}\right) \subset \bigcap_{\nu \geq 1} g^{* \nu}\left(A^{*}\right)=\left\{O^{* *}\right\} .
$$

For a pair of topological spaces $(X, Y)$, we shall mean by $X \Subset Y$ that $X$ is a relatively compact subset of $Y$.

LEMMA 5. There exists a strongly plurisubharmonic function $\varphi$ on an open neighborhood $W$ of $O^{* *}$ and a positive constant $c_{0}$ satisfying the following conditions: Put $D_{c}=\{z \in W: \varphi(z)<c\}$. Then, for any $c$, $0<c \leqq c_{0}$,

(i) $D_{c} \Subset W$,

(ii) $D_{c} \subset g^{*-1}\left(D_{c}\right)$,

(iii) $g^{*}: g^{*-1}\left(D_{c}-\left\{O^{* *}\right\}\right) \rightarrow D_{c}-\left\{O^{* *}\right\}$ is biholomorphic, and

(iv) $D_{c}$ is biholomorphic to an open ball in $C^{2}$.

Proof. Let $\left(W_{0},\left(w_{1}, w_{2}\right)\right)$ be a system of local coordinates such that $w_{1}\left(O^{* *}\right)=w_{2}\left(O^{* *}\right)=0$. Take a small neighborhood $W$ of $O^{* *}$ in $W_{0}$ such that $g^{* \nu}(W) \subset W_{0}$ for all large integers $\nu$. This is possible by Lemma 4. In terms of the coordinates $\left(w_{1}, w_{2}\right)$, we express $g^{*}$ locally by the Taylor series:

$$
\left\{\begin{array}{l}
w_{1}^{\prime}=a_{11} w_{1}+a_{12} w_{2}+h_{12}(w)+h_{13}(w)+\cdots \\
w_{2}^{\prime}=a_{21} w_{1}+a_{22} w_{2}+h_{22}(w)+h_{23}(w)+\cdots,
\end{array}\right.
$$

where $a_{i j} \in C$, and $h_{j k}(w)$ denotes the homogeneous $k$-th term of the $j$-th component of $g^{*}$. We put 


$$
T=\left(\begin{array}{ll}
a_{11} & a_{12} \\
a_{21} & a_{22}
\end{array}\right) .
$$
1.

LEMMA 6. The absolute value of all eigenvalues of $T$ are less than

Proof. Since $\left\{g^{*^{\nu}}\right\}$ is uniformly bounded, there is a subsequence $\left\{g^{*^{\nu} j}\right\}$ which converges to 0 uniformly on a relatively compact neighborhood $W_{1}$ of $O^{* *}$ in $W$. This implies that $\lim _{j \rightarrow+\infty} T^{\nu j}=0$. Then the lemma follows easily.

LEMMA 7. If the absolute values of all eigenvalues of $T$ are less than 1 , then there are a positive definite $2 \times 2$ hermitian matrix $H$ and a positive constant $\varepsilon$ such that

$$
(1-\varepsilon)(w, H w) \geqq(T w, H T w)
$$

for every $w \in C^{2}$, where $(w, z)=w_{1} z_{1}+w_{2} z_{2}, w=\left(w_{1}, w_{2}\right), z=\left(z_{1}, z_{2}\right)$.

The proof is easy.

In (7), we put

$$
F^{j}(w)=\sum_{k=2}^{\infty} h_{j k}(w) \quad(j=1,2)
$$

and

$$
F(w)={ }^{t}\left(F^{1}(w), F^{2}(w)\right) .
$$

SchWARZ's Lemma. On a small neighborhood $W_{1}$ of $O^{* *}$,

$$
\left|F^{j}(w)\right| \leqq K_{j}\|w\|^{2}, \quad\|w\|^{2}=\left|w_{1}\right|^{2}+\left|w_{2}\right|^{2},
$$

for some positive constants $K_{j}(j=1,2)$.

For a proof, see [1].

LEMMA 8. Let $H$ be as in Lemma 7. Then there exist positive numbers $c_{0}$ and $\varepsilon$ such that the equality

$$
(1-\varepsilon)(w, H w) \geqq\left(g^{*}(w), H g^{*}(w)\right)
$$

holds for any $c$ with $0 \leqq c \leqq c_{0}$, and any $w$ with $(w, H w) \leqq c$.

Proof. By the equality $g^{*}(w)=T w+F(w)$, we have

$$
\begin{aligned}
\left(g^{*}(w), H g^{*}(w)\right) & =(T w+F(w), H(T w+F(w))) \\
& \leqq(T w, H T w)+(F(w), H F(w))+2|(F(w), H T w)| \\
& \leqq(1-\varepsilon)(w, H w)+(F(w), H F(w))+2|(F(w), H T w)| .
\end{aligned}
$$

By Schwarz's Lemma, there are a positive constant $M$ and a small 
neighborhood $W_{2}$ of $O^{* *}$ such that

$$
|(F(w), H F(w))| \leqq M\|w\|^{4},
$$

and

$$
2|(F(w), H T(w))| \leqq M\|w\|^{3}
$$

for all $w \in W_{2}$. Put

$$
D_{b}=\{w \in W:(w, H w)<b\} .
$$

If we choose $c_{1}>0$ to be sufficiently small, then $D_{c_{1}} \subset W_{2}$, and, for $w \in D_{c_{1}}$, we have

$$
M\left(\|w\|^{4}+\|w\|^{3}\right) \leqq(\varepsilon / 2)(w, H w) .
$$

This implies that, for any $c, 0 \leqq c \leqq c_{1}$, and for any $w \in D_{c}$, the equality

$$
\left(g^{*}(w), H g^{*}(w)\right) \leqq(1-\varepsilon / 2)(w, H w)
$$

holds. Thus the proof of Lemma 8 is complete.

Now we go back to the proof of Lemma 5. Put $\varphi(w)=(w, H w)$. Then (i) and (iv) are clear. Note that $g^{*}: Z^{*}(\delta) \rightarrow D^{*}(\delta)=\lambda^{-1}(D(\delta))$ is proper and bimeromorphic. Since $\operatorname{dim} Z^{*}(\delta)=2$, there is a finite number of points $a_{1}, \cdots, a_{s}$ in $D^{*}(\delta)$ such that

$$
g^{*}: Z^{*}(\delta)-g^{*^{-1}}\left(\left\{a_{1}, \cdots, a_{s}\right\}\right) \rightarrow D^{*}(\delta)-\left\{a_{1}, \cdots, a_{s}\right\}
$$

is biholomorphic. Hence we can choose $c_{1}$ so that (iii) holds for all $c$ with $0<c \leqq c_{1}$. Since (iii) holds, $g^{*^{-1}} \mid D_{c}$ is biholomorphic near the strongly pseudoconvex boundary $\partial D_{c}$. Therefore to prove (ii), it is sufficient to show that $g^{*}\left(\partial D_{c}\right) \subset D_{c}$. Take any point $w \in \partial D_{c}$. Since $w \neq 0$, we have

$$
\left(g^{*}(w), H g^{*}(w)\right) \leqq(w, H w)-\varepsilon(w, H w)<(w, H w)=c
$$

by Lemma 8. Hence $g^{*}(w) \in D_{c}$.

We fix positive numbers $\alpha$ and $\beta$ such that $0<\alpha<\beta<c_{0}$. We consider the following subsets in $Z^{*}(\delta)$ :

$$
\begin{aligned}
& D_{\alpha}=\{w \in W: \varphi(w)<\alpha\}, \\
& D_{\beta}=\{w \in W: \varphi(w)<\beta\}, \\
& Q=\{w \in W: \alpha<\varphi(w)<\beta\}, \\
& G=g^{*^{-1}}\left(D_{\beta}\right), \text { and } \\
& P=g^{*^{-1}}(Q) .
\end{aligned}
$$

Here $\beta-\alpha$ is assumed to be small enough so that $\bar{Q} \cap \bar{P}=\varnothing$. Note that $g^{*} \mid P: P \rightarrow Q$ is biholomorphic by Lemma 5 (iii). Hence, taking 
infinitly many copies $\left(G-\bar{D}_{\alpha}\right)_{j}, j \in Z$, of $G-\bar{D}_{\alpha}$, we can form a complex manifold

$$
\widetilde{S}^{*}=\bigcup_{j}\left(G-\bar{D}_{\alpha}\right)_{j}
$$

by identifying each point $x \in P_{j}$ with $g^{*}(x) \in Q_{j-1}$, where $P_{j}$ and $Q_{j}$ denote the subsets of $\left(G-\bar{D}_{\alpha}\right)_{j}$ corresponding to $P$ and $Q$, respectively. We define a holomorphic automorphism $\widetilde{g}^{*}$ of $\widetilde{S}^{*}$ by

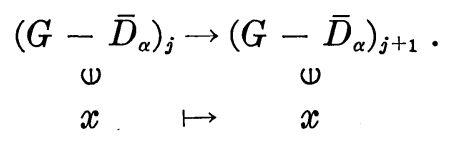

Then the orbit space

$$
S^{*}=\widetilde{S}^{*} /\left\langle\widetilde{g}^{*}\right\rangle
$$

is obviously a surface with a GSS by Lemma 5 (iv).

Lemma 9. There exists a bimeromorphic holomorphic mapping of $S$ onto $S^{*}$.

Proof. We define a holomorphic mapping

$$
\widetilde{\sigma}: \widetilde{S} \rightarrow \widetilde{S}^{*}
$$

as follows. For any point $p \in \widetilde{S}$, there is an integer $\nu$ such that $\widetilde{g}^{\nu}(p) €$ $E(\delta)$. Since $E(\delta)$ has no singular points, $\lambda: \lambda^{-1}(E(\delta)) \rightarrow E(\delta)$ is biholomorphic. Hence $\lambda^{-1} \circ \widetilde{g}^{\nu}(p)$ is a point in $Z^{*}(\delta)$. We find a non-negative integer $\mu$ such that $g^{* \mu} \circ \lambda^{-1} \circ \widetilde{g}^{\nu}(p) \in G-\bar{D}_{\alpha}$. Let $\tilde{i}: G-\bar{D}_{\alpha} \rightarrow\left(G-\bar{D}_{\alpha}\right)_{0} \subset$ $\widetilde{S}^{*}$ be the natural inclusion. Now we define

$$
\tilde{\sigma}(p)=\widetilde{g}^{*^{-\mu-\nu}} \circ \tilde{i} \circ g^{*^{\mu}} \circ \lambda^{-1} \circ \widetilde{g}^{\nu}(p) .
$$

As we see by this definition of $\tilde{\sigma}, \tilde{\sigma}$ is holomorphic provided that $\tilde{\sigma}$ is well-defined. Suppose that $\widetilde{g}^{\nu^{\prime}}(p) \in E(\delta)$ and $g^{* \mu^{\prime}} \circ \lambda^{-1} \circ \widetilde{g}^{\nu^{\prime}}(p) \in G-\bar{D}_{\alpha}$. It suffices to show

$$
\widetilde{g}^{*^{-\mu \prime-\nu \prime}} \circ \tilde{i} \circ g^{* \mu^{\prime}} \circ \lambda^{-1} \circ \widetilde{g}^{\nu^{\prime}}(p)=\widetilde{g}^{*^{-\mu-\nu}} \circ \tilde{i} \circ g^{* \mu} \circ \lambda^{-1} \circ \widetilde{g}^{\nu}(p) \text {. }
$$

First we suppose that $\nu^{\prime}=\nu$ and $\mu^{\prime}>\mu$. Then (10) is reduced to

$$
\tilde{i} \circ g^{*^{\kappa}}(q)=\tilde{g}^{* \kappa} \circ \tilde{i}(q),
$$

where $\kappa=\mu^{\prime}-\mu$ and $q=g^{* \mu} \circ \lambda^{-1} \circ \widetilde{g}^{\nu}(p) \in G-\bar{D}_{\alpha}$. Since both $q$ and $g^{* \kappa}(q)$ are in $G-\bar{D}_{\alpha}$, we have $\kappa=1$. Then (11) follows from the definition of $\widetilde{g}^{*}$. Next we suppose that $\nu^{\prime}>\nu$. Since both $\widetilde{g}^{\nu^{\prime}}(p)$ and $\widetilde{g}^{\nu}(p)$ are in $E(\delta)$, we have $\nu^{\prime}=\nu+1$. Hence (10) is reduced to

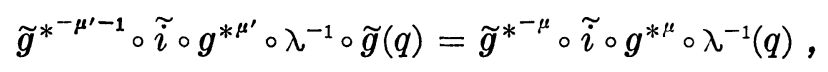


where $q=\widetilde{g}^{\nu}(p)$ and $\widetilde{g}(q)$ are in $E(\delta)$. Since both $g^{* \mu^{\prime}} \circ \lambda^{-1} \circ \widetilde{g}(q)$ and $g^{* \mu} \circ$ $\lambda^{-1}(q)$ are in $G-\bar{D}_{\alpha}$ and since $g^{* \mu^{\prime}} \circ \lambda^{-1} \circ \widetilde{g}(q)=g^{* \mu^{\prime}+1} \circ \lambda^{-1}(q)$, we have $\mu=\mu^{\prime}, \mu=\mu^{\prime}+1$, or $\mu=\mu^{\prime}+2$. If $\mu=\mu^{\prime}$, then (12) is reduced to

$$
\tilde{g}^{*^{-1}} \circ \tilde{i} \circ g^{*}(r)=\tilde{i}(r), \quad r=g^{*^{\mu} \circ \lambda^{-1}(q),}
$$

which follows from the definition of $\tilde{g}^{*}$. If $\mu=\mu^{\prime}+1$, (12) holds trivially. If $\mu=\mu^{\prime}+2$, then (12) is reduced to

$$
\tilde{g}^{*} \circ \tilde{i}(r)=\tilde{i} \circ g^{*}(r), \quad r=g^{*^{\mu-1}} \circ \lambda^{-1}(q),
$$

which follows also from the definition of $\widetilde{g}^{*}$. Hence (10) is proved. Consequently, $\tilde{\sigma}$ is well-defined. It is not difficult to see that $\tilde{\sigma}$ is generally one-to-one and satisfies $\tilde{\sigma} \circ \widetilde{g}=\widetilde{g}^{*} \circ \widetilde{\sigma}$. Hence $\widetilde{\sigma}$ defines a bimeromorphic holomorphic mapping

$$
\sigma: S=\widetilde{S} /\langle\widetilde{g}\rangle \rightarrow S^{*}=\widetilde{S}^{*} /\left\langle\widetilde{g}^{*}\right\rangle .
$$

Since $S^{*}$ contains a GSS and $S$ is a modification of $S^{*}$ by Lemma 9 , $S$ itself has a GSS. Therefore we have the following.

Proposition 2. If $O^{*} \in A$, then $S$ contains a GSS.

Proof of Theorem. The "if part" of the theorem is explained in Remark 2. The "only if part" of the theorem is clear by Propositions 1 and 2.

q.e.d.

\section{REFERENCES}

[1] R. Gunning ANd H. Rossi, Analytic Functions of Several Complex Variables, PrenticeHall, 1965.

[2] MA. KATo, Some remarks on subvarieties of Hopf manifolds, A symposium on complex manifolds, Kokyu-roku 240 (Research Institute for Mathematical Sciences, Kyoto University) 1975, 64-87, Tokyo J. Math. Vol. 2, No. 1, 47-61 (1979).

[3] MA. Kato, On a characterization of submanifolds of Hopf manifolds, Complex Analysis and Algebraic Geometry (Baily and Shioda, eds.), Iwanami Shoten Publishers, 1977, 191-206.

[4] MA. KATo, Compact complex manifolds containing "global" spherical shells, I, in the Proc. of the Int. Sym. of Algebraic Geometry, Kyoto, 1977, Kinokuniya, Tokyo, 1978, 45-84.

[5] H. Rossi, Attaching analytic spaces to an analytic space along a pseudoconcave boundary, Proc. of the Conference on Complex Analysis, Springer-Verlag, Berlin-Heidelberg-New York, 1964, 242-256.

[6] H. Rossi, Homogeneous strongly pseudoconvex hypersurfaces, Rice Univ. Studies 59 (1973), No. 1, 131-145.

Department of Mathematics

SOPHIA UNIVERSITY

KIOI-CHO 7, CHIYODA-KU

TOKYO, 102 JAPAN 
COMPACT COMPLEX SURFACES

Present Address

Mathematisches Institut

DER UNIVERSITÄT BONN

WEGELERSTRASSE 10

D-5300 BONN

Federal Republic of Germany 
